\title{
Occurrence of Whimper Singularities
}

\author{
S. T. C. Siklos \\ Department of Astrophysics, University of Oxford, Oxford OX1 3RQ, England
}

\begin{abstract}
Homogeneous space-times (i.e. those admitting a three-parameter group of isometries) are studied using the Newman Penrose formalism. It is found that solutions containing horizons depend on two fewer parameters than the most general solution, so that horizons and the associated whimper singularities are not stable features of homogeneous space-times. In the vacuum case, there are just three two-parameter families with horizons, two of which are the NUT solutions and certain plane waves.
\end{abstract}

\section{Introduction}

Singularities in space-time are characterised by the existence of an incomplete curve, $\gamma(v)$, with $0 \ll v<v_{+}$(see [1-3]). In particular, the more physical types of singularity occur if components of the Riemann tensor fail to tend to finite limits when measured in a frame which is parallelly propagated along $\gamma(v)$; these are called curvature (or p.p.) ${ }^{1}$ singularities. In this case the space-time cannot be extended through the singular point in any reasonable way. Curvature singularities can be subclassified as either $c^{0}$ scalar (curvature) ${ }^{1}$ or $c^{0}$ non-scalar (intermediate). If $v_{+}$is a $c^{0}$ non-scalar singularity, all polynomial invariants of the Riemann tensor tend to finite limits as $v \rightarrow v_{+}$; or equivalently [4], there exists a (non-parallelly propagated) frame in which components of the Riemann tensor do tend to finite limits. If such a frame does not exist, or if some polynomial invariant of $R_{a b c d}$ is badly behaved, the singularity is $c^{0}$ scalar.

Although it is known [5] that general, physically reasonable space-times must become singular somewhere, very little is known about the type of singularity which occurs. One approach to this problem is to investigate the behaviour of all members of a restricted class of solutions: if then the less desirable types of singularity turned out to arise only in a small subclass of these solutions, one could more readily believe that they are not a general feature of realistic universe models.

1 The classification here is due to Ellis and Schmidt [1] and differs from that given by Ellis and King in [2]. To avoid confusion, the terminology of [1] will usually be accompanied in parentheses by the equivalent description from [2] 


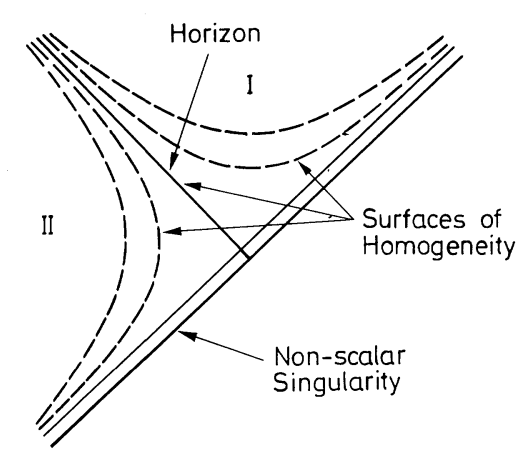

Fig. 1. A typical whimper in a homogeneous model. The broken lines represent surfaces of homogeneity, which are spacelike in region I, timelike in region II and null on the horizon $S_{0}$

The homogeneous ${ }^{2}$ models lend themselves particularly to this treatment, because the relevant equations can be studied using the techniques of ordinary differential equations. These models contain a one-parameter family of homogeneous hypersurfaces, $\boldsymbol{S}(u)$, local properties of which have been investigated in [6-8]. More recently, their global structure has come under scrutiny (see [2] for a systematic treatment), revealing some interesting features. It sometimes happens that the normals to the surfaces $S(u)$ are timelike in certain regions, but spacelike in others, adjacent regions being separated by a null surface of homogeneity, $\boldsymbol{S}_{0}$, which is a local isometry horizon. This structure will generally be accompanied by a $c^{0}$ non-scalar curvature singularity, as can be seen by the following argument. Firstly, there exists a frame on $S_{0}$ in which all components of the Riemann tensor are finite, because these components must be constant on $S_{0}$ in a group-invariant tetrad. However, for non-degenerate horizons, such a tetrad is related to a tetrad parallel along the null geodesic generators of $S_{0}$ by a Lorentz transformation which must diverge somewhere on $\boldsymbol{S}_{0}$ (see $[2,9]$ ). Unless the Weyl tensor is algebraically special, and the Ricci tensor is also of a specialised type, components of $R_{a b c d}$ will diverge in the parallelly propagated frame. The generators of $S_{0}$ will therefore encounter a $c^{0}$ non-scalar (intermediate) singularity. The causal structure of the singularity appears to be that of a null curve or surface (see Fig. 1). When the Weyl and Ricci tensors do have the necessary specialised forms, or when the horizon is degenerate, the null hypersurface may be complete; however, these possibilities can be eliminated by imposing reasonable energy conditions (see $\S 4$ ).

Non-scalar curvature singularities can also occur in spatially homogeneous solutions in the absence of a null surface of homogeneity, as for example in the Collinson-French solution [10]. In this case the space-time has the same conformal structure as a $k=-1$ Friedmann universe, but the initial singularity is $c^{0}$ non-scalar. Thus, although the term "whimper" was used in [2] to refer to any intermediate singularity, we shall apply it only to those which are accompanied by a horizon, as described above.

2 By "homogeneous" we mean "admitting a three-parameter isometry group simply transitive on hypersurfaces which are almost everywhere non-null" 
The purpose of this paper is to show that, contrary to the claim made in [2], (but in agreement with [11]), whimpers are not a general feature of homogeneous models. This supports the view that the singularities one is likely to encounter in the real universe are of the intuitive type, involving infinite matter density or pressure. The proof depends on determining the number of degrees of freedom in the initial value problem for whimper models, and comparing this with the corresponding number for the general homogeneous solution. It is found that whimper models usually allow two fewer degrees of freedom.

In fact, this result is not unexpected. For, consider the case of an empty spacetime. (The addition of matter fields is actually a straightforward generalisation of the vacuum case.) It is well-known [5] that the Cauchy development of a spacelike hypersurface, $S\left(u_{0}\right)$, is determined by four arbitrary functions on $S\left(u_{0}\right)$. In the homogeneous case, these functions are constant, so that the general solution depends on four parameters. In order to determine which of these solutions admits a horizon, it is necessary to examine initial data on a null hypersurface. This problem has been tackled by Penrose [12] and also by Sachs [13], who found that it suffices to prescribe just two functions over a null cone, or on two intersecting null hypersurfaces (plus data concerning the geometry of their intersection). When one hypersurface is homogeneous, the two functions become parameters as before, but these do not determine the development. However, it turns out that the additional requirement that the entire space-time be homogeneous is enough to fix conditions on the other hypersurface. (In other words, of all solutions which have constant initial data on one characteristic hypersurface, only a two-parameter family has a spatially homogeneous development.)

Although a number of different formalisms for homogeneous solutions have been developed in the literature $([7,14]$, for example) none is suitable for dealing with the problem described above. In most cases, this is because the tetrad is tied too closely to the invariant hypersurfaces, and is not well defined on the horizon. Indeed, the expansion of the normal congruence becomes infinite on this hypersurface, as do the quantities $a^{A}$ and $n^{A B}$ according to usual definitions [6], so it is clear that a simple application of the standard methods will produce anomalous results (cf. [15] with regard to [16]). Nor is it convenient to use a fluidbased tetrad as in [2], firstly because we shall want to consider vacuum solutions, and secondly because the whimper structure does not depend essentially on the fluid. (In the "exact" models of [2], any invariant vector field defined by the Riemann tensor could be used to replace the fluid flow lines.)

To avoid these difficulties we use a null tetrad, the scaling of which does not depend on the hypersurface normals. By choosing one vector to be tangent to a null geodesic which crosses the horizon, we ensure that the tetrad and Riemann tensor components are well-behaved on and near $\boldsymbol{S}_{0}$. This choice of tetrad is more akin to that of Newman and Penrose [17] than to an orthonormal tetrad, so rather than produce a new formalism along the lines of [7], we shall simply take over the familiar equations of [17].

The outline of this paper is as follows. In $\S 2$ the application of the NP formalism to homogeneous solutions is described. Slightly more detail is given than is needed here, because the same methods are used in a forthcoming paper [10] to determine the global properties of certain algebraically special solutions. 
The characterisation of the different Bianchi groups by means of the NP spin coefficients is given in $\S 3$. The method and characterisation follows closely that of Ellis and MacCallum [6], although some modification is required to deal with the null hypersurface. $\S 4$ contains a generalisation of a theorem given in [2], (Theorem 4.1), which states that whimpers cannot occur in universe models invariant under a group of class A. One might think that this result is due to some intrinsic property of the class A groups which prevents them acting on a manifold with metric signature $(o,-,-)$; however this is not the case. For, given any threeparameter group, one could choose a metric of this signature at one point in the group manifold and left translate it over the entire manifold yielding a $G_{3}$ invariant manifold with a degenerate metric, as required. In fact the exclusion of class A whimpers depends on an energy condition (a strong version of which is assumed in [2]) which we derive explicitly in Theorem 1 of $\S 4$. It is shown that class A models can only have horizons with expansion-free null generators, and that this situation cannot occur if certain conditions on the energy momentum tensor are fulfilled. (It is well-known [5] that realistic forms of matter cause null geodesics to focus.)

Finally, the results discussed above concerning initial null hypersurface data are derived in $\S 5$ for vacuum solutions and in $\S 6$ for perfect fluid. The method used is somewhat different from either [12] or [13], in that we calculate the degrees of freedom in terms of spin coefficients, rather than Riemann tensor or metric tensor components. The analogous results for initial spacelike hypersurfaces are easily obtainable, and are quoted in Appendix B, while Appendix A contains the necessary NP equations, with appropriate simplifications.

Sign and other conventions are those of Newman and Penrose [17], except that coordinate indices are lower case Latin, tetrad indices are Greek, and indices referring to an invariant triad are upper case Latin.

\section{Formalism}

Let $(\mathscr{M}, \boldsymbol{g})$ be a space-time with the usual analyticity properties (see e.g. [2]) which admits an isometry group $\boldsymbol{G}_{3}$ acting simply transitively on a family of space-like hypersurfaces, $\boldsymbol{S}(u)$. We shall use a $\boldsymbol{G}_{3}$-invariant null tetrad chosen in the following way. A single curve with null tangent vector $l^{a}$ is selected and then "dragged" over each $S(u)$ by the action of the group to define an invariant null congruence on $\mathscr{M}$ :

$$
\mathfrak{£}_{\xi_{A}} l^{a}=0 \quad A=1,2,3
$$

where $\xi_{A}$ are three linearly independent Killing vectors. A second null vector field, $n^{a}$, is defined invariantly and uniquely by requiring the unit normal to the hypersurfaces, $u^{a}$, to be expressible in the form

$$
u^{a}=\left(e^{\eta} n^{a}+e^{-\eta} l^{a}\right) / \sqrt{2} ; \quad u^{a} u_{a}=1
$$

for some $\eta=\eta(u) . n^{a}$ and $l^{a}$ satisfy the NP normalisation $l^{a} n_{a}=1$. The two complex vectors of the tetrad, $m^{a}$ and $\bar{m}^{a}$, are chosen at one point in each hypersurface to satisfy

$$
m^{a} \bar{m}_{a}=-1, \quad m^{a} m_{a}=0=m^{a} l_{a}=m^{a} n_{a} .
$$


They are also dragged over the hypersurfaces by the action of the group, and the normalisations (2.2) are maintained because Lie propagation preserves the metric. The tetrad is now uniquely defined, given the choice of $l^{a}$, except for a spatial rotation:

$$
m^{a} \rightarrow m^{a} e^{i \theta} ; \quad \theta=\theta(u) .
$$

The invariant scalar $e^{2 \eta}$ is a measure of the hyperbolic angle of tilt between the hypersurface normals and the null congruences. Its precise value depends on the normalisation of $l^{a}$, and therefore has no particular significance unless $l^{a}$ is determined by the Riemann tensor or its derivatives. However, under a change of normalisation

$$
l^{a} \rightarrow A l^{a}, \quad n^{a} \rightarrow A^{-1} n^{a}
$$

the transformation $e^{-2 \eta} \rightarrow A^{-2} e^{-2 \eta}$ follows from the definition (2.1), so that the sign of $e^{-2 \eta}$ is independent of the normalisation. It will be seen that the field equations can be written in a form which depends on $e^{-2 \eta}$ (rather than $e^{-\eta}$ ), so that in certain cases, the null congruences can be continued into regions where $e^{-2 \eta}$ is negative. In these regions, the real vector field $\sqrt{2} e^{-\eta} u^{a} \equiv e^{-2 \eta} l^{a}+n^{a}$ is spacelike and therefore defines a family of "timelike" hypersurfaces. Furthermore, when $e^{-2 \eta}=0$, the hypersurface is null. The sign of $e^{-2 \eta}$ therefore indicates, for any choice of $l^{a}$, the nature of the invariant hypersurfaces.

The tangent spaces to $S(u)$ at each point are spanned by the vectors $E_{A}^{a}$ :

$$
\begin{aligned}
& E_{1}^{a}=\left(e^{-\eta} l^{a}-e^{\eta} n^{a}\right) / \sqrt{2}, \quad E_{2}^{a}=\left(m^{a}+\bar{m}^{a}\right) / \sqrt{2}, \\
& E_{3}^{a}=\left(m^{a}-\bar{m}^{a}\right) / \sqrt{2} i
\end{aligned}
$$

(In fact many expressions involving $\boldsymbol{E}_{\boldsymbol{A}}$ could be simplified by choosing $\boldsymbol{E}_{2}$ and $\boldsymbol{E}_{3}$ to be complex conjugates. Despite this, we shall use the triad (2.5) in order to maintain a resemblance with previous approaches.) Using the usual notation for derivatives along the tetrad directions [17], we have for any $G_{3}$-invariant function $\varphi(u)$ :

$$
\boldsymbol{E}_{A}(\varphi)=0 \Rightarrow \delta \varphi=\bar{\delta} \varphi=\left(e^{-\eta} D-e^{\eta} \Delta\right) \varphi=0 .
$$

The rotation coefficients of the null tetrad are defined by the commutators of the tetrad vectors:

$$
\begin{aligned}
& {[\Delta, D]=(\gamma+\bar{\gamma}) D+(\varepsilon+\bar{\varepsilon}) \Delta-(\tau+\bar{\pi}) \bar{\delta}-(\bar{\tau}+\pi) \delta} \\
& {[\delta, D]=(\bar{\alpha}+\beta-\bar{\pi}) D+\varkappa \Delta-\sigma \bar{\delta}-(\bar{\varrho}+\varepsilon-\bar{\varepsilon}) \delta} \\
& {[\delta, \Delta]=-\bar{v} D+(\tau-\bar{\alpha}-\beta) \Delta+\bar{\lambda} \bar{\delta}+(\mu-\gamma+\bar{\gamma}) \delta} \\
& {[\bar{\delta}, \delta]=(\bar{\mu}-\mu) D+(\bar{\varrho}-\varrho) \Delta-(\bar{\alpha}-\beta) \bar{\delta}+(\alpha-\bar{\beta}) \delta}
\end{aligned}
$$

and these quantities are easily shown to be constant on the surfaces of homogeneity (cf. [6]). By applying the commutators (2.7) in turn to an arbitrary invariant scalar, and using the relations (2.6) one obtains the following useful 
identities :

$$
\begin{aligned}
D e^{-2 \eta} & =-(\gamma+\bar{\gamma})-(\varepsilon+\bar{\varepsilon}) e^{-2 \eta}, \\
0 & =x e^{-2 \eta}+(\bar{\alpha}+\beta-\bar{\pi}), \\
0 & =-\bar{v}+(\tau-\bar{\alpha}-\beta) e^{-2 \eta}, \\
0 & =(\bar{\mu}-\mu)+(\bar{\varrho}-\varrho) e^{-2 \eta} .
\end{aligned}
$$

The equations governing the propagation of the rotation coefficients are the 18 complex NP equations ((a)-(r) in [17]) which are listed with appropriate specialisation in Appendix A. They comprise 16 (real) Jacobi identities for the null tetrad (cf.. [6]) and the defining equations for the 20 independent components of the Riemann tensor. They imply the Bianchi identities, which will be designated (B1)-(B11), with reference to the order given in [18], and also the identities (2.8)(2.11). We shall avoid using the Bianchi identities, except when the equivalent derivation via the NP equations is excessively cumbersome. For this reason only (B2) is given in Appendix A.

The shear and expansion of the hypersurface normals are easily obtainable from the decomposition of $l_{a ; b}$, etc. given in $[10]$ :

$$
\begin{aligned}
\vartheta_{a b} \equiv & u_{a ; b}=\sqrt{2} \operatorname{Re}\left\{\left(\gamma e^{\eta}-\bar{\varepsilon} e^{-\eta}\right) E_{1(a} E_{2 b)}-2 \sqrt{2}(\bar{\alpha}+\beta) E_{1(a} m_{b)}\right. \\
& \left.+\left(\bar{\sigma} e^{-\eta}-\lambda e^{\eta}\right) m_{a} m_{b}-\left(\mu e^{\eta}-\varrho \bar{e} e^{-\eta}\right) m_{a} \bar{m}_{b}\right\}
\end{aligned}
$$

which is symmetric by virtue of Equation (2.11);

$$
\vartheta=\sqrt{2} \operatorname{Re}\left\{(\varepsilon-\bar{\varrho}) e^{-\eta}+(\mu-\bar{\gamma}) e^{\eta}\right\} .
$$

\section{Classification of Bianchi Groups}

Three parameter Lie groups can be classified into 10 types, according to a scheme described in [6]. Generally, these types are determined from the commutator functions $\gamma_{B C}^{A}$ of an invariant basis such as (2.5)

$$
\left[E_{A}, E_{B}\right]=E_{C} \gamma_{A B}^{C}
$$

The commutator functions transform as mixed tensors under automorphisms of the vector space tangent to each invariant hypersurface. The group action is characterised by the invariants of $\gamma_{B C}^{A}$ under these transformations, namely the rank and signature of the pseudotensor $n^{A B}=\frac{1}{2} \varepsilon^{C D(A} \gamma_{C D}^{B)}$, and the vanishing or non-vanishing of the covector $a_{B}=\frac{1}{2} \gamma_{B A}^{A}$. These quantities must satisfy

$$
n^{A B} a_{B}=0
$$

which are equivalent to the Jacobi identities $\varepsilon^{A B C}\left[\boldsymbol{E}_{A},\left[\boldsymbol{E}_{B}, \boldsymbol{E}_{C}\right]\right]=0$. One solution, $a_{B}=0$, characterises groups in class A; all other solutions describe class B groups. Details of the classification are given in Appendix B. The components of $n^{A B}$ and 
$a_{B}$ in the basis (2.5) are expressible in terms of the spin coefficients as follows:

$$
\begin{aligned}
& \sqrt{2} a_{1}=e^{\eta} \operatorname{Re}\left\{\left(\varrho e^{-2 \eta}+\mu\right)\right\}, \\
& \sqrt{2} a_{2}=\operatorname{Re}\{(\tau-\bar{\pi}+\bar{\alpha}-\beta)\}, \\
& \sqrt{2} a_{3}=\operatorname{Re}\{-i(\tau-\bar{\pi}+\bar{\alpha}-\beta)\}, \\
& \sqrt{2} n^{11}=e^{-n} \operatorname{Re}\{2 i(\bar{\varrho}-\varrho)\} \\
& \sqrt{2} n^{12}=\operatorname{Re}\{i(\bar{\pi}-\tau+\bar{\alpha}-\beta)\}, \\
& \sqrt{2} n^{13}=\operatorname{Re}\{(\bar{\pi}-\tau+\bar{\alpha}-\beta)\}, \\
& \sqrt{2} n^{23}=e^{n} \operatorname{Re}\left\{\left(\sigma e^{-2 \eta}+\bar{\lambda}\right)\right\}, \\
& \sqrt{2} n^{22}=e^{\eta} \operatorname{Re}\left\{i(\bar{\sigma}+\varrho-2 \varepsilon) e^{-2 \eta}+i(\lambda+\bar{\mu}-2 \bar{\gamma})\right\}, \\
& \sqrt{2} n^{33}=e^{\eta} \operatorname{Re}\left\{i(\bar{\sigma}-\varrho+2 \varepsilon) e^{-2 \eta}+i(\lambda-\bar{\mu}+2 \bar{\gamma})\right\} .
\end{aligned}
$$

These are obtained by means of the commutators (2.7), using also the relations (2.8)-(2.11). The criteria for the group to be in class A are therefore

$$
\operatorname{Re}\left\{\mu+\varrho e^{-2 \eta}\right\}=0 \quad \text { or } \quad \mu+\varrho e^{-2 \eta}=0
$$

and

$$
\tau-\bar{\pi}+\bar{\alpha}-\beta=0 \quad \text { or } \quad \tau-2 \beta-x e^{-2 \eta}=0
$$

the alternative forms following from (2.9) and (2.11).

The expressions (3.2) and (3.3) may be considerably simplified by judicious use of the remaining freedom in the null tetrad, i.e. choice of $l^{a}$ at one point on each hypersurface, and also the rotation (2.3). They could of course be reduced to their canonical forms as described in [6], but this does not prove very useful in the present context. In this paper, we shall usually require $l^{a}$ to be tangent to a family of null geodesics $(\chi=0)$, and use the scaling (2.4) and (2.3) to set $\varepsilon=0$. This still leaves the freedom to choose $l^{a}$ and make an invariant rotation (2.3) on one hypersurface, but the most convenient choice depends on the group type.

It is worth examining a few such choices to illustrate the role of the different spin coefficients, and also to establish a connection with the more familiar approaches to homogeneous cosmological models. When $x=0$, Equation (3.5) becomes $\tau=2 \beta$, which can always be satisfied at $u=u_{0}$ by choice of $l^{a}$. The NP equations (c), (e) and (k) then show that this condition holds for all $u$ :

$$
D(\tau-2 \beta)=\bar{\varrho}(\tau-2 \beta)+\sigma(\bar{\tau}-2 \bar{\beta}) .
$$

(If the group is in class A, (3.5) is of course automatically satisfied.) Except in Types VIII and IX, we may use the freedom to set $n^{11}=n^{12}=n^{13}=0$ at $u=u_{0}$, i.e. $\varrho-\bar{\varrho}$ $=\bar{\beta}-\alpha=0$. Then NP equations (a) and (d), (e) and (k) again show that these conditions hold everywhere despite the tetrad restriction $x=0$ :

$$
\begin{aligned}
& D(\varrho-\bar{\varrho})=\varrho^{2}-\bar{\varrho}^{2} \\
& D(\bar{\alpha}-\beta)=(\bar{\alpha}-\beta) \varrho+\bar{\sigma}(\alpha-\bar{\beta}) .
\end{aligned}
$$


(If the group is in class B and $\tau=2 \beta$, these conditions hold by virtue of the Jacobi identities (3.1).) We then have $v=\mu-\bar{\mu}=0$, from (2.10) and (2.11). These remarks have the following significance. Each quantity $b^{A}$ defines a space-time vector $b^{A} E_{A}^{a}$ and also a unique null vector, the hypersurface projection of which is $b^{A} E_{A}^{a}$. In each of the cases considered above $n^{A B}$ has an eigenvector with vanishing eigenvalue (this is just $a_{B}$ for class B groups); furthermore, the tetrad is chosen so that the associated null vector is a multiple of $l^{a}$. We may conclude that such eigenvectors determine two geodesic $(\varkappa=v=0)$, hypersurface orthogonal $(\varrho-\bar{\varrho}=\mu-\bar{\mu}=0)$ invariant null congruences in all group types except VIII and IX.

It is easily shown, using NP equations $(\mathrm{d}, \mathrm{e}, \mathrm{o}, \mathrm{r})$, that when the matter is such that

$$
\Phi_{10} e^{-\eta}+\Phi_{21} e^{\eta}=0
$$

(e.g. for vacuum and non-tilted perfect fluid) and the tetrad is chosen as above $(\tau=2 \bar{\alpha}=2 \beta=\bar{\pi}, \varrho=\bar{\varrho}$, in Types I-VII) the spin coefficient $\alpha$ must vanish (except for group Type $\mathrm{VI}_{-1 / 9}$, which allows an extra family of solutions with $\alpha \neq 0$ - see Appendix B). This means that the entire tetrad is parallelly propagated along the $l^{a}$ congruence $(\varepsilon=\pi=0)$. The solutions then possess symmetries analogous to the well-known axisymmetric stationary solutions [19], (i.e. the group contains an invertible abelian subgroup [20]), and the line element can be written in the simple form

$$
d s^{2}=e^{-2 \eta}\left(d \tau^{2}-d z^{2}\right)-a(\tau) g_{\alpha \beta}(\tau) w^{\alpha} w^{\beta} ; \quad \alpha, \beta=1,2
$$

where $w^{1}, w^{2}, d z$ are invariant $l$-forms and $\operatorname{det}\left(g_{\alpha \beta}\right)=1$. In vacuum or non-tilted perfect fluid with a stiff equation of state, $a(\tau)$ may be found explicitly, and its form for each group type is listed in Appendix B.

For the remainder of this paper we shall want to consider the possibility of the surfaces of homogeneity becoming null. With the choice of tetrad described in $\S 2$, this can only happen if $e^{-\eta}=0^{3}$, in which case $n^{a}$ is parallel to the hypersurface normal. When this occurs the criteria for the different group types have to be modified slightly because the vectors (2.5) are not defined in the limit $e^{-\eta}=0$. Note also that the shear (2.12), expansion (2.13) and some components of $\gamma_{B C}^{A}$ diverge in this limit, so that results for the null hypersurface obtained from equations containing these quantities must be interpreted with caution. One way to avoid this difficulty is to consider the space-time tensor field $C_{a b}^{\alpha}$ defined uniquely by

$$
\tilde{E}_{\beta[a ; b]}=C_{a b}^{d} \tilde{E}_{\beta d} \quad \beta=0-3
$$

for the four vector fields $\left\{\tilde{E}_{\beta}\right\}=\left\{\tilde{E}_{0}, \tilde{E}_{A}\right\}$ :

$$
\tilde{E}_{0}^{a}=l^{a}, \quad \tilde{E}_{1}=e^{-\eta} E_{1}, \quad \tilde{E}_{2}=E_{2}, \quad \tilde{E}_{3}=E_{3} .
$$

The vector and pseudo-tensor fields $a_{b}$ and $n^{a b}$ are defined by a decomposition analogous to that for $\gamma_{A B}^{D}$ :

$$
a_{b}=\frac{1}{2} C_{b a}^{a} ; \quad n^{a b}=\frac{1}{2}\left(e^{-2 \eta} l_{d}+n_{d}\right) \eta^{\operatorname{def}(a} C_{e f}^{b)} .
$$

3 The other possibility, $e^{+\eta}=0$, becomes $e^{-\eta}=0$ when $n^{a}$ and $l^{a}$ are interchanged 
Since all the vector fields involved are smooth even when $e^{-2 \eta}=0$, these quantities are well-defined everywhere. The Jacobi identity (3.1) is equivalent to

$$
a_{b} n^{a b}=0 \text {. }
$$

To retrieve expressions such as (3.2) and (3.3), one simply projects $C_{a b}^{d}$ into the spacelike hypersurface using $\boldsymbol{E}_{A}$ :

$$
a_{A}=E_{A}^{b} a_{b}, \quad n^{A B}=E_{a}^{A} E_{b}^{B} n^{a b} .
$$

Alternatively, one can use the invariant triad $\tilde{E}_{A}$ to obtain the quantities $\tilde{a}_{A}, \tilde{n}^{A B}$ which remain finite when $e^{-2 \eta}=0$, and can be used equivalently to characterise the different group types. The components of $\tilde{n}^{A B}$ are just those given in (3.3), with the factor preceding the curly bracket omitted. In terms of the null tetrad, $a_{b}$ is given by

$$
a^{b}=(\mu+\bar{\mu}) l^{a}-(\varrho+\bar{\varrho}) n^{a}-(\tau-\bar{\pi}+\bar{\alpha}-\beta) \bar{m}^{a}-(\bar{\tau}-\pi+\alpha-\bar{\beta}) m^{a}
$$

so that on $S_{0}$, the criteria for class A groups, which is the vanishing of $\tilde{E}_{A}^{b} a_{b}$ becomes

$$
\mu+\bar{\mu}=0 \quad \text { and } \quad \tau-\bar{\pi}+\bar{\alpha}-\beta=0 .
$$

Note that for a null hypersurface the significant part of $a^{b}$ (i.e. excluding the component in the normal direction, which depends on the scaling of the tetrad $\tilde{E}_{\alpha}$ ) does not lie in the hypersurface tangent space. In choosing the null tetrad on $S_{0}$ for class B solutions, we shall use $a^{b}$ to determine the $l^{b}$ direction, since the normal now lies in the hypersurface, and can be used to define one of the invariant triad vectors.

\section{Horizons in Homogeneous Models}

With the choice of tetrad described in $\S 2$, a null surface of homogeneity occurs only when $e^{ \pm 2 \eta}=0$. We shall assume that $e^{-2 \eta}=0$ on $S_{0}$, so that $n^{a}$ lies in, and is normal to $S_{0}$, and

$$
\Delta \varphi=0=\delta \varphi \quad \text { on } \quad \boldsymbol{S}_{0}
$$

for any $\boldsymbol{G}_{3}$-invariant scalar $\varphi(u)$. We shall also choose $l^{a}$ to be tangent to a family of null geodesics which crosses $\boldsymbol{S}_{0}$. The null hypersurface is supposed to consist of regular points of the space-time, so in this tetrad all the spin coefficients and Riemann tensor components are well-behaved. Since $n^{a}$ is null and invariant, there exists for each null generator, $\lambda$, of $S_{0}$ a Killing vector field $\xi^{a}(\lambda)$ which on $\lambda$ is parallel with, and commutes with, $n^{a}$. It follows that $\xi^{a}(\lambda)$ may be chosen such that $\xi^{a}=n^{a}$ on $\lambda$, defining a local isometry horizon. When the Killing vector field is the same for each $\lambda$, i.e. $n^{a}=\xi^{a}$ everywhere on $S_{0}$, the hypersurface is called a Killing horizon. A necessary condition for this is $\lambda=\mu+\bar{\mu}=\nu=0$ on $S_{0}$, so that according to (3.10), Killing horizons can occur in either class A or class B solutions.

On $\boldsymbol{S}_{0}, \gamma+\bar{\gamma}$ (related to the "surface gravity") cannot be made to vanish by means of an invariant scale change, since under the transformation (2.4) we have

$$
(\gamma+\bar{\gamma}) \rightarrow A^{-1}(\gamma+\bar{\gamma})-\Delta A^{-1}
$$


Local isometry horizons therefore fall into two classes; they are either degenerate or non-degenerate, depending on the vanishing or non-vanishing of $(\gamma+\bar{\gamma})$. When $(\gamma+\bar{\gamma})=0$ the null generators of $\boldsymbol{S}_{0}$ are complete, as is the geodesic congruence normal to the non-null surfaces of homogeneity. However, the horizon can be reached from any space-time point by travelling a finite affine distance along a null geodesic congruence, so that extensions similar to those given in [20] for the Robinson-Bertotti solution can be made. When $(\gamma+\bar{\gamma}) \neq 0$, the null Killing vector has a fixed point on $\lambda$ [9], which will not usually be a regular space-time point. For, suppose $k^{a}$ is tangent to $\lambda$ and satisfies $k_{a ; b} k^{b}=0$. Then $n^{a}=e^{-(\gamma+\bar{\gamma})} k^{a}$ and $e^{-(\gamma+\bar{\gamma}) v}=(\gamma+\bar{\gamma}) s$, where $s$ is affine along $\lambda$ and $v$ is the ignorable coordinate corresponding to $\xi^{a}$. Each point in the spacelike 2-surface in $\boldsymbol{S}_{0}$ for which $s=0$ is invariant under the action of $\xi^{a}$, and hence fixed. As $s \rightarrow 0$, tetrads based on $k^{a}$ (and in particular, tetrads parallel along $\lambda$ ) diverge from the invariant null tetrad based on $n^{a}$, so that some components of the Riemann tensor, which are constant in the latter tetrad, will generally diverge in the former. When this happens, the null hypersurface generators run into a $c^{0}$ non-scalar singularity. Otherwise, the Riemann tensor on $\boldsymbol{S}_{0}$ may be invariant under boosts in the $n^{a}$ direction (i.e. when it is $D$-specialised [27] $)$, in which case the fixed points are $c^{0}$ regular. An analysis similar to that given by Boyer [9] for Killing horizons then shows that the horizon is bifurcate, as in the NUT solutions.

It will be apparent from the examination in $\S 5$ of initial data on $S_{0}$ that these types of horizon can all occur in homogeneous models. However, if one insists on realistic energy conditions, solutions with $\mu(\gamma+\bar{\gamma})=0$ on the horizon can be eliminated:

Theorem 1. Killing horizons, degenerate isometry horizons and class $A$ whimpers are incompatible with the null convergence condition

$$
2 \Phi_{22} \equiv R_{a b} n^{a} n^{b}>0 .
$$

Proof. We use an invariant null tetrad with $n^{a}$ parallel to the hypersurface normal on $S_{0}$ and $l^{a}$ tangent to a family of null geodesics. We have $x=0$ and also

$$
\nu=\mu-\bar{\mu}=\bar{\alpha}+\beta-\bar{\pi}=0
$$

from (2.9)-(2.11). Then NP equation (n) becomes

$$
\Phi_{22}+\mu^{2}+\mu(\gamma+\bar{\gamma})+\lambda \bar{\lambda}=0 .
$$

Clearly the condition (4.2) cannot be satisfied if either $(\gamma+\bar{\gamma})=0$, as on degenerate horizons or if $\mu=0$, as in class A solutions, and on Killing horizons.

\section{Initial Value Problem for Vacuum Whimpers}

By solving the characteristic initial value problem on $S_{0}$ we shall establish

4 When the Riemann tensor is rather less specialised ("[211]-specialised") the "surface" $s=0$ may be $c^{0}$-regular for the generators of $\boldsymbol{S}_{0}$, but $c^{0}$-singular for the congruence normal to $\boldsymbol{S}(u)$. Since one is interested in the behaviour of the normal congruence, we shall not distinguish between this complicated situation and the straightforward singular case 
Theorem 2. The only vacuum homogeneous ${ }^{5}$ space-times with horizons are

(i) A two-parameter family of homogeneous plane waves given by

$$
d s^{2}=2 d u d v-2 d z d \bar{z}+H d u^{2}
$$

with $H=\operatorname{Re}\left\{c z^{2} u^{2 i(1-x)}\right\}$ and $0<x<2 c$. These admit group Types $V I I_{h}, V I_{h}$ and $I V$ when $c \leqq \varkappa<2 c, 0<\varkappa<c$ and $\varkappa=c$ respectively (see [10]);

(ii) a two-parameter family of Type $V I_{-1 / 9}$ solutions;

(iii) the two-parameter family of NUT solutions, of Type IX,VIII or II (see $[6,22])$.

Proof. This is not a genuine initial value problem, because data on one null hypersurface is not sufficient to determine a unique solution of the vacuum field equations. We shall therefore need a criterion which picks out, from amongst all solutions with homogeneous initial data, those which are also homogeneous away from the initial hypersurface. Accordingly, we start with a vacuum solution $(\mathscr{M}, \boldsymbol{g})$ of the Einstein equations, a NP tetrad defined everywhere in $\mathscr{M}$, and a null hypersurface $S_{0}$ in $\mathscr{M}$, with tangent space spanned by $n^{a}, m^{a}+\bar{m}^{a},\left(m^{a}-\bar{m}^{a}\right) / i$. We then assume that the tetrad can be chosen such that.

(a) On $S_{0}$ the tetrad spin coefficients are constant [i.e. they satisfy (4.1)] and Equations (2.10) and (2.11) hold with $e^{-2 \eta}=0$.

(b) The tetrad is such that $\varepsilon=0$, and Equation (2.9) with $\chi=0$ is satisfied everywhere in $\mathscr{M}$. Condition (a) is clearly a necessary condition for $\boldsymbol{S}_{0}$ to be homogeneous, while Condition (b) can always be satisfied in a homogeneous space-time by using the tetrad gauge freedom. The set of solutions satisfying these conditions will therefore include all whimper models. To prove the theorem we shall show that any member of this set is uniquely determined by the values of two (real) spin coefficients on $S_{0}$ and furthermore, that the solution thus defined is homogeneous. The identification of the solutions then follows from a close examination of the constraint equations.

It is convenient to use the following notation: $\Gamma_{i}$ represents the spin coefficients of the null tetrad; $\Psi_{i}$ and $\Phi_{i j}$ are the Weyl and Ricci tensor tetrad components (the latter are zero in this section); and $D^{n}$ is the $n^{\text {th }} l^{a}$-directional derivative operator which acts on these quantities. All are evaluated on the null hypersurface $S_{0}$. The problem reduces firstly to determining $D^{n} \Gamma_{i}$ from $\Gamma_{i}$, since then the spin coefficients can be computed close to $S_{0}$ by means of a Taylor expansion along the $l^{a}$ geodesic congruence, and secondly to finding the constraints on $\Gamma_{i}$.

In vacuum, the NP equations divide into three groups on $S_{0}$ : equations (a)-(i) are propagation equations, defining 18 of the $24 D \Gamma_{i}$ in terms of $\Gamma_{i}$ and $\Psi_{i}$; equations $(\mathrm{j})-(\mathrm{m})$ involve no (non-zero) derivatives, and so define 8 of the $10 \Psi_{i}$; and equations (n)-(r) are constraint equations for $\Gamma_{i}$. In order to find the missing $\Psi_{i}$, namely $\Psi_{0}$, we resort to the Bianchi identity B 2 , which in vacuum, becomes

$$
\Delta \Psi_{0}=(4 \gamma-\mu) \Psi_{0}-2(2 \tau+\beta) \Psi_{1}+3 \sigma \Psi_{2} .
$$

5 See Footnote 2. Solutions with the Kantowski-Sachs symmetry [28] are not included in this definition. However, the only vacuum solutions of this type are "Schwarzschildian" [21], and are derivable from the NUT solutions 
This fails to express $\Psi_{0}$ in terms of $\Gamma_{i}$ only when $4 \gamma-\mu=0$, which cannot happen according to (4.4) unless $\lambda=\mu=\gamma=0$. Fortunately it emerges, when the constraint equations are solved at the end of this section, that this possibility does not exist in non-flat vacuum solutions. Of the $D \Gamma_{i}$, all but $D \varkappa, D \varepsilon$ and $D \pi$ are determined by the propagation equations. However, since $x$ and $\varepsilon$ are identically zero throughout $M$ by choice of tetrad, while $D \pi=D(\alpha+\bar{\beta})$ from (2.9), all the $D \Gamma_{i}$ can be written in terms of $\Gamma_{i}$ and $\Psi_{i}$ and hence in terms of just $\Gamma_{i}$.

Furthermore, by operating on each equation with $D^{n-1}$, and applying the commutator relation (2.8), one obtains $D^{n} \Gamma_{i}$ in terms of $\Gamma_{i}$ as required. (The $n^{\text {th }}$ derivative of $(5.1)$ determines $D^{n} \Psi_{0}$ unless $(4+2 n) \gamma=\mu$, and equality here need not be considered for the same reasons as before.)

To demonstrate that the values of $D^{n} \Gamma_{i}$ thus obtained represent a consistent vacuum solution, we shall show that the only constraints on $\Gamma_{i}$ are imposed by NP equations (n)-(r). The derivatives of equations (a)-(m) and also of (2.9) cannot give rise to constraints since they are automatically satisfied by definition of $D^{n} \Gamma_{i}$, while Equation (2.8) serves to define a function $e^{-2 \eta}$, chosen to vanish on $\boldsymbol{S}_{0}$. Nor do the derivatives of equations $(n)-(r)$ provide additional constraints, because identities of the form $D C_{i} \equiv \sum_{j} A_{i j} C_{j}$ are readily established for each constraint equation $C_{i}=0$. Similarly, derivatives of the right hand side of Equation (2.10) and (2.11) vanish when the original equations are satisfied. Finally, we observe that the Bianchi and Jacobi identities (3.9) need not be considered: the former are derived from derivatives of the NP equations, while the latter are equivalent to $((r)+(o)-(m))$, and $\operatorname{Im}((q)-(l))$. The values of $\Gamma_{i}$, subject to the constraint equations (n)-(r) are therefore sufficient to determine a unique development. It is apparent that the development consists of a family of homogeneous hypersurfaces, and that the vector field $e^{-2 \eta} l^{a}+n^{a}$ is normal to these surfaces.

There are 14 algebraically independent constraints on $\Gamma_{i}$, i.e. Equations (2.9)(2.11), (o)-(r) and $\operatorname{Re}(n)$, so this leaves 10 of the 24 spin coefficients undetermined. Of these $4(x$ and $\varepsilon$ ) are known by virtue of the choice of tetrad, while 4 more combinations can be reduced to zero, using the remaining tetrad freedom on $S_{0}$. This comprises a constant null rotation about $n^{a}$ :

$$
n^{a} \rightarrow n^{a}, m^{a} \rightarrow m^{a}+\bar{B} n^{a}, l^{a} \rightarrow l^{a}+B m^{a}+\bar{B} \bar{m}^{a}+B \bar{B} n^{a},
$$

the rotation (2.3) and the boost (2.4), all at one point of $S_{0}$. We therefore expect to find two-parameter families of solutions.

To complete the proof of Theorem 2, we now need only to examine the constraint equations to see what possibilities can arise. After substitution for $\Psi_{i}$ and $\pi$, these equations become

$$
\begin{aligned}
& 0=\mu^{2}+\lambda \bar{\lambda}+\mu(\gamma+\bar{\gamma}), \\
& 0=(\tau-\bar{\alpha}-2 \beta) \gamma+\bar{\gamma} \beta+(\tau+\beta) \mu+\alpha \bar{\lambda}, \\
& 0=\sigma(\mu-3 \gamma+\bar{\gamma})+\bar{\lambda} \varrho+\tau(\tau+\beta-\bar{\alpha}), \\
& 0=2 \mu \varrho-\gamma \bar{\varrho}-\bar{\gamma} \varrho+\alpha \bar{\alpha}+\beta \bar{\beta}-2 \alpha \beta-\tau(\bar{\beta}-\alpha-\bar{\tau}), \\
& 0=\mu(2 \alpha+\bar{\beta})+\lambda(\bar{\alpha}-2 \beta+\tau)-\gamma(\bar{\beta}-\bar{\tau})-\bar{\gamma} \alpha, \\
& 0=\mu-\bar{\mu}=v .
\end{aligned}
$$


We first dismiss the cases mentioned above, when $\mu=\gamma=\lambda=0$. For then $\tau=\bar{\alpha}-\beta=0$, from (5.5) and (5.6) and hence [using NP equations (j), (l) and (m)] $\Psi_{4}=\Psi_{3}=\Psi_{2}=0$. If we now choose $l^{a}[$ by means of (5.2)] to be a principal null direction of the Weyl tensor, so that $\Psi_{0}=0$, Equation (5.1) gives $\Psi_{1}=0$, either directly, or if $\beta=0$ via equation $(\mathrm{k}){ }^{6}$ The spacetime is then flat. (It is easy to convince oneself, using the Bianchi identities for an invariant tetrad with $\Psi_{0}=0$ everywhere, that the only vacuum homogeneous spacetime with $\Psi_{i}=0$ on $S_{0}$ is Minkowski space.)

The class A solutions are characterised by $\tau=2 \beta$ and $\mu=0$. Under the null rotation (5.2), $\sigma$ transforms according to

$$
\sigma \rightarrow \sigma+\bar{B}(\tau+2 \beta)+\bar{B}^{2}(\mu+2 \gamma)+\bar{B}^{3} v
$$

and may always be made to vanish on $S_{0}$ (since $\gamma=0$ gives flat space-time, as above). It is then found that the only possible solution has $\gamma \bar{\varrho}+\bar{\gamma} \varrho=0$, with all other spin coefficients zero. Substitution into the modified form of $\tilde{n}^{A B}$ described at the end of $\S 3$ reveals that the solutions are invariant under groups of Types II, VIII or IX. Using the remaining freedom (2.4) to set $\gamma \bar{\gamma}=1$ leaves a two-parameter family of solutions which is readily identified as the NUT family [22, 10].

Class B solutions divide into two sets, depending on whether $a^{b}$ is essentially spacelike or null on $S_{0}$. This is determined by the vanishing or non-vanishing of $\mu$. ( $\mu$ is invariant under the transformation (5.2), since $v=0$.)

When $\mu=0$, but $\tau-2 \beta \neq 0$, Equations (5.3), (5.4) and (5.7) imply $\lambda=\gamma-\bar{\gamma}$ $=\tau-\bar{\alpha}-\beta=0$. By setting $\Psi_{0}=0$ as before, ${ }^{6}$ one obtains a solution of Type III (i.e. $\mathrm{VI}_{-1}$ ), with $\alpha+\bar{\beta}=\sigma=0$. This is not unexpected, since it is known [6] that the Type VIII NUT solutions also admit a group of Type III. By comparing the actual values of the spin coefficients of the exact solution [10] with those of the initial value solution, one can check that the only class B solutions with $\mu=0$ on $S_{0}$ are just these NUT solutions.

When $\mu \neq 0$, the transformation (5.2) may be used essentially to line up $l^{b}$ and $a^{b}$, by setting $\tau-2 \beta=0$. Equations (5.4) and (5.7) yield

$$
\beta \lambda+\bar{\beta}(3 \mu+\gamma-\bar{\gamma})=0
$$

which leads to two families of solutions. One possibility has $\lambda=1$ and $\gamma, \mu$ as the only non-zero spin coefficients, which are related by (5.3). These are the plane wave solutions of Theorem 2 [10]. Alternatively, Equation (5.9) can be satisfied if $9 \mu^{2}-(\gamma-\bar{\gamma})^{2}=\lambda \bar{\lambda}$, and this also leads to a two-parameter family. They are of Type Bbii (i.e. $\mathrm{VI}_{h}$ with $h=-1 / 9$ ) [6] but the corresponding exact solutions are not known explicitly.

\section{Perfect Fluid Whimpers}

The characteristic initial value problem for matter filled homogeneous solutions is not fundamentally different from the vacuum problem described in $\S 5$. The tetrad freedom is the same and the same NP equations become propagation equations for $\Gamma_{i}$ on $S_{0}$, but the number of constraint equations is reduced as equations (n)-(r) are required to evaluate $\Phi_{i j}$ from $\Gamma_{i}$. Generally, there will be only six constraints on $\Gamma_{i}$, arising from the three Jacobi identities and (2.10) and (2.11), but others may arise when relations between the $\Phi_{i j}$ are given by a specific choice of

6 See Note Added in Proof 
matter type and equation of state. Various $\Phi_{i j}$ components, as well as $\Psi_{0}$, may not be immediately obtainable from the NP equations, but this depends on the precise form of the matter fields. In very exceptional cases the problem is not soluble.

To be specific, we shall consider an isentropic perfect fluid, for which

$$
R_{a b}=-(\mu+p) v_{a} v_{b}+\frac{1}{2}(\mu-p) g_{a b} ; \quad v_{a} v^{a}=1 .
$$

The tetrad components of the Ricci tensor are

$$
\begin{aligned}
& \Phi_{00}==\frac{1}{2}(\mu+p) v_{1}^{2} \quad \Phi_{11}=\frac{1}{4}(\mu+p) \quad\left(v_{1} v_{2}+v_{3} \bar{v}_{3}\right) \quad \Phi_{22}=\frac{1}{2}(\mu+p) v_{2}^{2} \\
& \Phi_{01}=\frac{1}{2}(\mu+p) v_{1} v_{3} \quad \Phi_{12}=\frac{1}{2}(\mu+p) v_{2} v_{3} \quad \Phi_{02}=\frac{1}{2}(\mu+p) v_{3}^{2} \\
& \Lambda=1 / 24(\mu-3 p)
\end{aligned}
$$

where

$$
v^{a}=v_{2} l^{a}+v_{1} n^{a}-\bar{v}_{3} m^{a}-v_{3} \bar{m}^{a} ; \quad 2 v_{1} v_{2}-2 v_{3} \bar{v}_{3}=1 .
$$

It is convenient to start with $\Gamma_{i}$ as initial data and determine $D^{n} \Gamma_{i}$ as in $\S 5$, rather than consider initial data for the matter fields, although the two methods are clearly equivalent.

We again assume that the conditions (a) and (b) of $\S 5$ hold on $S_{0}$, and show that the space-time is determined by certain of the $\Gamma_{i}$. As before these conditions must necessarily be satisfied if the solution is to be homogeneous. Of the 20 Riemann tensor components, only 14 can be evaluated from the NP equations, since 4 of the 18 non-propagation equations do not involve $\Psi_{i}$ or $\Phi_{i j}$. We must therefore determine $\Psi_{0}, \Phi_{00}$ and, say, $\Phi_{01}$ and $\left(\Phi_{11}-3 \Lambda\right)$ by other means. This is no problem for the perfect fluid (6.1), since $\left(\Phi_{11}-3 \Lambda\right)$ can be found in terms of $\left(\Phi_{11}+3 \Lambda\right)$ from the equation of state, while all $\Phi_{i j}$ are expressible as functions of $\Phi_{11}, \Phi_{12}$ and $\Phi_{22}$ using (6.1) and (6.2). As before, $\Psi_{0}$ is obtained from (B2), although the method is not quite so straightforward owing to the presence of the $D \Phi_{02}$ term. This must be evaluated using $D \Phi_{11}, D \Phi_{12}$ and $D \Phi_{22}$, since these derivatives can be expressed in terms of $\Phi_{i j}$ and $\Gamma_{i}$, and hence in terms of just $\Gamma_{i}$, by means of the differentiated NP equations [or equivalently, the Bianchi identities (B9), (B10) and (B11)]. This is possible because the relevant equations do not contain $D \sigma$, the only $D \Gamma_{i}$ which depends on $\Psi_{0}$. Having found $\Psi_{0}$ from (B2), equation (b) gives $D \sigma$, so that all $D \Gamma_{i}$ and hence by differentiation, all $D^{n} \Gamma_{i}$ may be found from $\Gamma_{i}$, as required.

To show that the values of $D^{n} \Gamma_{i}$ thus obtained describe a well-defined solution, one can simply apply the arguments used in the vacuum case. The only constraints on $\Gamma_{i}$ arise from equations (2.10) and (2.11); from the Jacobi identities $((r)+(o)-(m))$ and $\operatorname{Im}((l)-(q))$; and also from equating the values of $\Phi_{02}$ derived firstly from equation $(p)$ and secondly from the relations (6.1). [No analogous constraints are obtained from the two possible methods of evaluating $D^{n} \Phi_{02}$, because these are consistent when the relevant (differentiated) Bianchi identity is satisfied; in this case it is (B2) which is satisfied by definition of $D^{n-1} \Psi_{0}$ ]. This makes a total of eight constraints, which together with the four remaining degrees of tetrad freedom and the fact that $\varepsilon, x$ and $\pi$ are known, leaves six $\Gamma_{i}$ 
undetermined. We should therefore expect perfect fluid whimper solutions to depend in general on six parameters.

A closer examination of the constraint equations indeed reveals that for each of the most general group Types (IX, VIII, VII, VI) there is a six-parameter families of solutions, which broadly speaking generalise the vacuum solutions (i) and (ii) of Theorem 2 by the addition of matter fields. The other family of vacuum solutions (iii) does not generalise in this way, since it occurs only for special matter types (see Appendix B). Whimper solutions exist for all other group types except I, which does not allow tilted fluids [7]. The important point is that such solutions depend on two fewer parameters than the general tilted fluid model of the same group type, so Whimper models, as defined in $\S 2$ are unstable in the set of all homogeneous solutions.

\section{Appendix A}

The NP equations (a)-(r) in [17] and the Bianchi identity B2 of [18] are given below, as appropriate for the invariant tetrad described in $\S 2$. They have $\varepsilon=\chi=\delta=0$.
(a) $D \varrho=\left(\varrho^{2}+\bar{\sigma} \sigma\right)+\Phi_{00}$
(b) $D \sigma \quad=(\varrho+\bar{\varrho}) \sigma+\Psi_{0}$
(c) $D \tau-\Delta \chi=(\tau+\bar{\pi}) \varrho+(\bar{\tau}+\pi) \sigma+\Phi_{01}+\Psi_{1}$
(d) $D \alpha=\varrho \alpha+\beta \bar{\sigma}+\varrho \pi+\Phi_{10}$
(e) $D \beta=(\alpha+\pi) \sigma+\bar{\varrho} \beta+\Psi_{1}$
(f) $D \gamma=(\tau+\bar{\pi}) \alpha+(\bar{\tau}+\pi) \beta+\tau \pi+\Psi_{2}-\Lambda+\Phi_{11}$
(g) $D \lambda=(\varrho \lambda+\bar{\sigma} \mu)+\pi^{2}+(\alpha-\bar{\beta}) \pi+\Phi_{20}$
(h) $D \mu=(\bar{\varrho} \mu+\sigma \lambda)+\pi \bar{\pi}-\pi(\bar{\alpha}-\beta)+\Psi_{2}+2 \Lambda$
(i) $\quad D v-\Delta \pi=(\pi+\bar{\tau}) \mu+(\bar{\pi}+\tau) \lambda+(\gamma-\bar{\gamma}) \pi+\Psi_{3}+\Phi_{21}$
(j) $\Delta \lambda=-(\mu+\bar{\mu}) \lambda-(3 \gamma-\bar{\gamma}) \lambda$ $+(3 \alpha+\bar{\beta}+\pi-\bar{\tau}) v-\Psi_{4}$
(k) $\quad 0 \quad=\varrho(\bar{\alpha}+\beta)-\sigma(3 \alpha-\bar{\beta})+(\varrho-\bar{\varrho}) \tau-\Psi_{1}+\Phi_{01}$
(l) $0=(\mu \varrho-\lambda \sigma)+\alpha \bar{\alpha}+\beta \bar{\beta}-2 \alpha \beta+\gamma(\varrho-\bar{\varrho})-\Psi_{2}+\Lambda+\Phi_{11}$
(m) $0=(\varrho-\bar{\varrho}) v+(\mu-\bar{\mu}) \pi+\mu(\alpha+\bar{\beta})+\lambda(\bar{\alpha}-3 \beta)-\Psi_{3}+\Phi_{21}$
(n) $-\Delta \mu=\left(\mu^{2}+\lambda \bar{\lambda}\right)+(\gamma+\bar{\gamma}) \mu-v \pi+(\tau-3 \beta-\bar{\alpha}) v+\Phi_{22}$
(o) $-\Delta \beta=(\tau-\bar{\alpha}-\beta) \gamma+\mu \tau-\sigma \nu-\beta(\gamma-\bar{\gamma}-\mu)+\alpha \bar{\lambda}+\Phi_{12}$
(p) $-\Delta \sigma=(\mu \sigma+\bar{\lambda} \varrho)+(\tau+\beta-\bar{\alpha}) \tau-(3 \gamma-\bar{\gamma}) \sigma+\Phi_{02}$
(q) $\Delta p=-(\varrho \bar{\mu}+\sigma \lambda)+(\bar{\beta}-\alpha-\bar{\tau}) \tau+(\gamma+\bar{\gamma}) \varrho-\Psi_{2}-2 \Lambda$
(r) $\Delta \alpha=\varrho \nu-(\tau+\beta) \lambda+(\bar{\gamma}-\bar{\mu}) \alpha+(\bar{\beta}-\bar{\tau}) \gamma-\Psi_{3}$.
B2 $\Delta \Psi_{0}+D \Phi_{02}=(4 \gamma-\mu) \Psi_{0}-2(2 \tau+\beta) \Psi_{1}+3 \sigma \Psi_{2}-\lambda \Phi_{00}$

$$
+2(\bar{\pi}-\beta) \Phi_{01}+2 \sigma \Phi_{11}+\varrho \Phi_{02} \text {. }
$$




\section{Appendix B}

Details of the classification of three-parameter Lie algebras are summarised in the table below. The characterisation in terms of $\mathcal{N}=\operatorname{det}\left(n^{A B}\right)$, $N=\frac{1}{2}\left(n^{A B} n_{A B}-n^{2}\right), n=n_{A}^{A}$ and $a^{A}$ (see $\S 3$ ) is taken from [7]. Type $\mathrm{VI}_{h}$ and $\mathrm{VII}_{h}$ groups are parametrised by an invariant number $h$, defined by $h=-a^{A} a_{A} / N$.

At any fixed value of $u$, an invariant triad can be chosen which gives $n^{A B}$ the canonical form appropriate for its signature and rank, as listed in Columns 3 and 4 of the table (cf. [6]).

The integer $r$ in Column 5 represents the number of arbitrary parameters in the general vacuum solution of each group type. This is calculated by considering an initial data set for a spatially homogeneous solution, consisting of $\left(h_{A B}, \chi_{A B}, n^{A B}\right.$, $\left.a^{B}\right)$ given on a Cauchy surface. $\left(h_{A B} \chi_{A B}\right.$ are triad components of the first and second fundamental forms of the hypersurface, as in [14].) These 21 parameters completely determine the solution. However, this number is reduced by the 3 Jacobi identities, 4 constraint equations, the 9 dimensional field of automorphisms of the tangent space to the Cauchy surface, and finally the coordinate freedom $u \rightarrow u+u_{0}$, leaving just 4 essential parameters in the most general group Types $\left(\mathrm{VI}_{h}\right.$, VII $_{h}$, VIII and IX). In Types VI and VII, $h$ counts as one of the parameters, so that $r=3$ for each $h$. This includes the limiting values of $h=0$, as in $\mathrm{VI}_{0}$ and $\mathrm{VII}_{0}$, and $h= \pm \infty$ as in Type IV. These remarks also apply to Type III which is an alternative name for $\mathrm{VI}_{h}$ with $h=-1$. (It is sometimes treated as a separate group type because its derived algebra is one dimensional, while other $\mathrm{VI}_{h}$ groups have two dimensional derived algebras. This does not effect our calculations.) The one exceptional case occurs in $\mathrm{VI}_{h}$ when $h=-1 / 9$. For this group type, two of the constraint equations are degenerate in vacuum, as is most easily seen by comparing equations (analagous to) (4.2b) and (4.2c) of [14]. There is therefore an extra parameter in the solution, and $r=4$. For Types I and II, the requirements in Column 5 restrict the number of essential parameters in a straightforward way, giving respectively $r=1$ and $r=2$, while for Type $\mathrm{V}, r=1$ follows from a more careful study of the initial data set [23].

The number $r$ also describes the comparative degree of generality of nonvacuum solutions. For example, the numbers in the 7 th column of the table are the numbers of arbitrary parameters in perfect fluid homogeneous models with given equation of state. Usually, $s=r+4$, but in Types I and II the constraint equations restrict the form of the fluid (cf. [7]). Type $\mathrm{VI}_{-1 / 9}$ is not exceptional in general perfect fluid solutions, because the constraint equations become degenerate only for energy momentum tensors satisfying Equation (3.6).

The function in the final column relates to the line element (3.7), which describes a homogeneous solution with energy momentum tensor satisfying $T^{02}=T^{03}=0$ (these components refer to the pseudonormal invariant tetrad (2.5) which puts $C_{B C}^{A}$ in canonical form, as in [14]). When in addition $T^{00}=T^{11}, a(\tau)$ satisfies equations of the form (e.g. for Type $\mathrm{VII}_{h}$ )

$$
(\log a)^{\prime \prime}+\left((\log a)^{\prime}\right)^{2}-4=0 ; \quad, \equiv \frac{d}{d \tau},
$$

giving rise to the functions in Column 8 . 
Table 1. The classification and characterisation of three-parameter Lie groups. The quantities $r$ and $s$ describe the number of parameters in the general solution of each group type, for vacuum and for perfect fluid. The function $a(\tau)$ refers to the line element (3.7)

\begin{tabular}{|c|c|c|c|c|c|c|c|}
\hline $\begin{array}{l}\text { Group } \\
\text { type }\end{array}$ & $\begin{array}{l}\text { Group } \\
\text { class }\end{array}$ & $\operatorname{Sig}(n)$ & $\operatorname{Rank}(n)$ & Characterisation & $r$ & $s$ & $a(\tau)$ \\
\hline I & & 0 & 0 & $\mathscr{N}=N=n=0$ & 1 & 2 & \\
\hline II & & 1 & 1 & $\mathscr{N}=N=0$ & 2 & 5 & \\
\hline $\mathrm{VI}_{0}$ & $A$ & 0 & 2 & $\mathcal{N}=0 \quad N>0$ & 3 & 7 & $A \tau+B$ \\
\hline $\mathrm{VII}_{0}$ & $\left(a^{A}=0\right)$ & 2 & 2 & $\mathscr{N}=0 \quad N<0$ & 3 & 7 & \\
\hline VIII & & 1 & 3 & $\mathscr{N}<0$ & 4 & 8 & \\
\hline IX & & 3 & 3 & $\mathscr{N}>0$ & 4 & 8 & \\
\hline IV & & 1 & 1 & $\mathscr{N}=N=0$ & 3 & 7 & \\
\hline $\mathrm{V}$ & & 0 & 0 & $\mathscr{N}=N=n=0$ & 1 & 5 & $A e^{2 \tau}$ \\
\hline $\mathrm{VI}_{h}$ & $B$ & 0 & 2 & $\mathcal{N}=0 \quad N>0$ & 4 & 8 & $+B e^{-2 \tau}$ \\
\hline $\operatorname{VII}_{h}$ & $\left(a^{A} \neq 0\right)$ & 2 & 2 & $\mathscr{N}=0 \quad N<0$ & 4 & 8 & \\
\hline $\mathrm{VI}_{-1 / 9}^{n}$ & & 0 & 2 & $\mathcal{N}=0 \quad N=9 a^{A} a_{A}$ & 4 & 7 & \\
\hline
\end{tabular}

The general vacuum solutions for group Types I (Kasner [24]), II (Taub [25]) and V (Joseph [26]) are known, and illustrate some of the above results:

Kasner: $d s^{2}=\tau^{2 \gamma}\left(-d \tau^{2}+d z^{2}\right)+\tau\left(\tau^{2 \beta} d x^{2}+\tau^{-2 \beta} d y^{2}\right) \quad 4 \beta^{2}=4 \gamma+1$.

Taub: $\quad d s^{2}=g(\tau) \tau^{b^{2} / 8}\left(-d \tau^{2}+d z^{2}\right)+\tau\left(g(\tau) d x^{2}+1 / g(\tau)(d y-x d z)^{2}\right) \quad$ with $g(\tau)=\frac{1}{2}\left((a \tau)^{b}+(a \tau)^{-b}\right) \tau / b$.

Joseph:

$d s^{2}=\sinh 2 a \tau\left\{\left(-d \tau^{2}+d z^{2}\right)+(\tanh a \tau)^{1 / \sqrt{3}} e^{-2 a z} d y^{2}+(\tanh a \tau)^{-1 / \sqrt{3}} e^{-2 a z} d x^{2}\right\}$.

\section{References}

1. Ellis, G.F.R., Schmidt,B.G. : Singular space-times, submitted to G.R.G.

2. Ellis, G.F. R., King, A. R.: Commun. math. Phys. 38, 119 (1974)

3. Clarke, C.J.S., Schmidt, B. G. : G.R.G. 8, 129 (1977)

4. Siklos, S.T.C.: to be published

5. Hawking,S.W., Ellis, G.F.R.: The large scale structure of space-time. Cambridge: Cambridge University Press 1973

6. Ellis, G.F.R., MacCallum, M.A.H.: Commun. math. Phys. 12, 108 (1969)

7. King, A. R., Ellis, G.F. R. : Commun. math. Phys. 31, 209 (1973)

8. Collins, C. B., Ellis, G.F.R.: Singularities in Bianchi cosmologies. Preprint (1977)

9. Boyer, R. G. : Proc. Roy. Soc. Lond. 311, 245 (1969)

10. Siklos, S.T.C.: Preprint, Oxford University (1977)

11. Evans, A. B.: Phys. Lett. 55 A, 271 (1975)

12. Penrose, R.: Null hypersurface initial data for classical fields of arbitrary spin and for general relativity. Unpublished dissertation

13. Sachs, R. K.: J. Math. Phys. 3, 908 (1962)

14. Collins, C.B., Hawking, S.W.: Ap. J. 180, 317 (1973)

15. King, A. R.: Phys. Lett. 54 A, 115 (1975)

16. Collins, C. B.: Commun. math. Phys. 39, 131 (1974)

17. Newman,E., Penrose, R.: J. Math. Phys. 3, 566 (1962)

18. Pirani,F.A.E.: Lectures on general relativity, (eds. H. Bondi, F. A. E. Pirani, A. Trautman). Englewood Cliffs. New Jersey: Prentice Hall 1964

19. Reina, C., Treves, A.: G.R.G. 7, 817 (1976)

20. Carter,B.: Black hole equilibrium states. In: Black holes (eds. C. DeWitt, B. S. DeWitt). New York: Gordon and Breach 1973 
21. Ehlers, J., Kundt, W.: Gravitation: An introduction to current research (ed. L. Witten). New York: Wiley 1962

22. Siklos, S.T.C.: Phys. Lett. 59 A, 173 (1977)

23. Siklos, S.T.C.: Unpublished Ph.D. Thesis, Cambridge University (1976)

24. Kasner,E.: Trans. Am. Math. Soc. 27, 155 (1925)

25. Taub, A.H.: Ann. Math. 53, 472 (1951)

26. Joseph, V.: Proc. Cambridge Phil. Soc. 62, 87 (1966)

27. Clarke,C.J.S.: Commun. math. Phys. 41, 65 (1975)

28. Kantowski, R., Sachs, R.K.: J. Math. Phys. 7, 443 (1966)

Communicated by R. Geroch

Received October 18, 1977

Note Added in Proof. The assumption made in two different situations on p. 267, that $l^{a}$ can be chosen so that $\bar{\Psi}_{0}$ vanishes, needs some elaboration. $\bar{\Psi}_{0}$ can be made to vanish using (5.2) unless all the other Weyl tensor components are zero. In the first situation $(\mu=\gamma=\lambda=0)$ this ${ }^{3}$ can only happen if $\varrho$ and $\sigma$ are the only non-zero spin coefficients. (Note that the $l^{a}$-directional derivative of (B2) provides an additional constraint when $\gamma=0$.) One can easily verify that then $D^{n} e^{-2 \eta}=0$, so that the surfaces of homogeneity remain null and $S_{0}$ is not a horizon. In the second situation on p. 267 (with $\tau-\bar{\alpha}-\beta=0$ ) $\bar{\Psi}_{2}=0$ implies $\alpha=\bar{\beta}$ (from $N P$ equation $(l)$ ), contradicting the assumption that $\tau-2 \beta \neq 0$. 